\title{
The Kilopixel Array Pathfinder Project (KAPPa), a 16 pixel integrated heterodyne focal plane array
}

\author{
Christopher E. Groppi ${ }^{\mathrm{a}}$, ${ }$, Caleb H. Wheeler ${ }^{\mathrm{a}}$, Hamdi Mani ${ }^{\mathrm{a}}$, Patrick McGarey ${ }^{\mathrm{a}}$, Todd Veach $^{\mathrm{a}}$, \\ Sander Weinreb $^{\mathrm{b}}$, Damon Russell ${ }^{\mathrm{b}}$, Jacob W. Kooi ${ }^{\mathrm{b}}$, Arthur W. Lichtenberger ${ }^{\mathrm{c}}$, Christopher K. \\ Walker $^{\text {, }}$, Craig Kulesa ${ }^{\mathrm{d}}$ \\ ${ }^{a}$ Arizona State University, School of Earth and Space Exploration, PO Box 876004, Tempe, AZ, \\ USA 85287-6004; ${ }^{\mathrm{b}}$ California Institute of Technology, 1200 E. California Blvd., Pasadena, CA USA

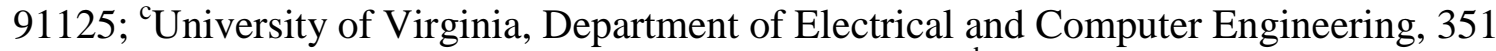

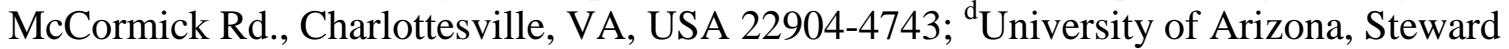 \\ Observatory, 933 N. Cherry Ave., Tucson, AZ, USA 85721.
}

\begin{abstract}
KAPPa (the Kilopixel Array Pathfinder Project) is developing key technologies to enable the construction of heterodyne focal plane arrays in the terahertz frequency regime with $\sim 1000$ pixels. The leap to $\sim 1000$ pixels requires solutions to several key technological problems before the construction of such a focal plane is possible. The KAPPa project will develop a small (16-pixel) 2D integrated heterodyne focal plane array for the $660 \mathrm{GHz}$ atmospheric window as a technological pathfinder towards future kilopixel heterodyne focal plane arrays.
\end{abstract}

Keywords: THz imaging, array receivers

\section{INTRODUCTION}

The THz spectral regime is one of the last frontiers in both remote sensing and detector development. At this confluence of infrared and radio techniques, two approaches are used to detect $\mathrm{THz}$ radiation. Direct detection techniques, similar to those used in the infrared, have been pushed to lower energies to detect $\mathrm{THz}$ photons. Photoconductors have been built for astrophysics applications that operate at wavelengths up to $200 \mu \mathrm{m}$ (e.g. [1], [2]). For longer wavelength (THz) detection, cryogenic TES bolometer arrays have been developing at a rapid rate during the last decade. The UK SCUBA2 instrument, with 10,240 pixels at two THz colors $(850 \mu \mathrm{m}$ and $450 \mu \mathrm{m})$ has been commissioned at the James Clerk Maxwell Telescope [3]. This instrument will likely revolutionize astrophysics in the same way its predecessor SCUBA (with 37 pixels at $850 \mu \mathrm{m}$ and 91 pixels at $450 \mu \mathrm{m}$ ) did a decade ago.

While direct-detection $\mathrm{THz}$ focal plane arrays are extremely valuable and have opened a new window on astrophysics, they do have fundamental limitations. Direct detectors do not preserve the relative phase information of arriving photons; therefore they deliver modest spectral resolution. Filters typically define $\sim 10 \%$ imaging bandwidths. FabryPerot and grating spectrometers can be constructed and placed in front of the incoherent arrays to disperse the incoming light, but offer modest spectral resolution $(\mathrm{R}<1000-10,000)$ and cannot spatially and spectrally multiplex simultaneously. They are therefore primarily used to image thermal emission from dust in astronomical sources. For astronomical and atmospheric applications, there is considerable interest in the behavior of gas species. To disentangle their motions, instruments with high spectral resolutions are required $(\mathrm{R}>10,000)$. In molecular clouds, this gas makes up 99\% of the material by mass, and is the dominant player in the physics of star and planet formation. Kinematic information extracted from spectra collected using coherent techniques allows the detailed study of gas dynamics, in addition to the chemical information collected from the detection of a particular gas species. Various gas species have emission lines in the terahertz wave-band that offer a huge wealth of information. They can be used to determine gas properties of the

*cgroppi@asu.edu; phone: +1-480-965-6436; fax: +1-480-965-8102; http://thz.asu.edu

Millimeter, Submillimeter, and Far-Infrared Detectors and Instrumentation for Astronomy VI, edited by Wayne S. Holland, Jonas Zmuidzinas, Proc. of SPIE Vol. 8452

84520Y @ 2012 SPIE · CCC code: 0277-786X/12/\$18 · doi: 10.1117/12.927358 
interstellar medium (ISM), such as mass, kinematics, temperature, density, and chemical composition. Large scale surveys of these lines both in the Milky Way and beyond can be used to help answer long standing questions in astrophysics, such as star formation, the life cycle of the ISM, and the chemical evolution of galaxies. As shown in figure 1, ISM structures like giant molecular clouds can subtend hundreds of square degrees on the sky, and have enormous spatial dynamic ranges spanning cloud size structures down to individual protostellar objects. Interferometers like ALMA, while incredibly sensitive, have tiny fields of view. This makes such instruments ill-suited for wide field imaging. Imaging these clouds requires focal plane arrays of coherent detectors on large telescopes to achieve the required spatial dynamic range while observing molecular gas line emission with sufficient spectral resolution. In astrophysics and remote sensing applications, the speed of imaging a given area of sky is directly proportional to the number of detectors. An increase of a factor of $\sim 1000$ in observing efficiency provided by a kilopixel array receiver would revolutionize observational sub- $\mathrm{mm} / \mathrm{THz}$ astronomy, opening up widefield imaging and dramatically increasing the scientific return of new and future facilities like CCAT and SPT. Other fields of astronomy went through a similar revolution some time ago. For example, infrared astronomy has graduated from single-pixel technology to arrays consisting of tens of thousands of detectors. The current state-of-the-art pixel count for coherent $\mathrm{THz}$ arrays is 64 pixels (i.e. SuperCam, [4]) or less. While these instruments are a great leap forward from single pixel detectors, the pixel count is still inadequate for wide field $\mathrm{THz}$ imaging of weak spectral lines. Two white papers submitted to the 2010 decadal survey emphasize the critical role that focal plane arrays will play in the future [5],[6]. These reports conclude that a significant effort must be made to develop key technologies before attempting to construct the ambitious and expensive kilopixel class instruments demanded by future scientific applications. KAPPa aims to develop several key technologies the Goldsmith and Kerr reports identify as essential for the construction of kilopixel arrays.

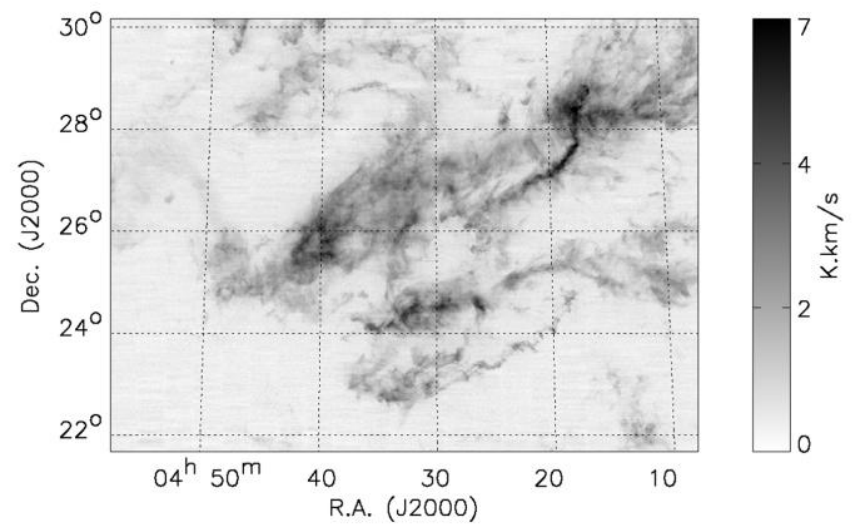

Figure 1. An integrated intensity image of the Taurus molecular cloud complex covering over 120 square degrees on the sky in the light of the ${ }^{13} \mathrm{CO}(1-0)$ transition made with the SEQUOIA 32 pixel coherent array [7],[8].

In this project, we are developing and testing several solutions to outstanding challenges that must be met if $\sim 1000$ pixel $\mathrm{THz}$ heterodyne arrays are to be realized. The cost and complexity of such an instrument demand a systematic approach to the solution of these technical challenges before attempting to construct a fully-featured science instrument. We are constructing a compact pathfinder instrument with a 16-pixel 2D integrated focal plane at $660 \mathrm{GHz}$ as a vehicle to test technical solutions. These solutions can then be applied to the design and construction of a kilopixel class array receiver.

\section{ARRAY ARCHITECTURE}

\subsection{Challenges for Kilopixel Array Development}

During the design phase for SuperCam, it became apparent that the expansion of the 0D paradigm of stacking independent mixers in the focal plane would become overwhelmingly complicated and unreliable for $\sim 100$ pixel arrays. Similarly, experience with SuperCam has shown the authors that the 1D integration of SuperCam will also become unwieldy at pixel counts exceeding $\sim 100$. We have identified several key areas where technological development is essential to the construction of larger format arrays. These areas and their potential solutions are listed below.

- Mechanical and Electrical Complexity: Larger split-block based 1D integrated arrays will become too unwieldy to fabricate. Separate interconnect sets are still needed for each linear array.

Solution: Use 2D integration. 
- Economical and fast fabrication techniques for waveguide and feeds: CNC micromachining techniques can make large amounts of waveguide accurately, quickly and economically. Waveguide feedhorns remain difficult to manufacture, especially in large numbers.

Solution: Use a custom tool to directly drill efficient feedhorns in metal.

- RF and DC interconnects, wire count, and complexity: At pixel counts of $\sim 1000$, individual coaxial transmission lines for each IF signal become overwhelmingly complex and creates an untenable cryogenic heat transfer situation.

Solution: Multi-conductor DC cabling with adequate wire count already exists. Multi-conductor RF ribbon cable and connectors with integral heat sinking should be implemented.

- LO power availability, multiplexing and injection: Kilopixel SIS arrays will require power-combining solidstate multiplier based sources to produce enough LO power.

Solution: Several techniques exist for multiplexing LO power and efficiently injecting that power (e.g. phase gratings, Si Etalons)

- Magnetic field for SIS devices: 1000 individually controllable electromagnets are unwieldy and too complicated.

Solution: Use carefully engineered high field permanent magnets.

\subsection{Pathfinder Focal Plane Array Design}

KAPPa is designed to take advantage of the packaging simplification offered by a $2 \mathrm{D}$ integrated configuration. In this architecture, all pixels of the array are fabricated in a single, vertically stacked module. A lower "detector block" houses the SIS mixer chips, waveguide backshort, magnets, Low Noise Amplifier (LNA) and associated IF circuitry and all DC bias circuitry. The KAPPa detector block will be machined from a single piece of 145 copper alloy, with each pixel cell fabricated on an elevated pedestal. A circuit board with holes machined for each pedestal will route IF and DC lines (see figure 2). To simplify IF line routing, each microstrip will be brought to the back side of the board through a via and then sent to another plane with Corning Gilbert G3PO connectors. These connectors will directly mate to the IF flex circuit, or to a fan-out board if required. DC lines will be routed on the front face of the mixer circuit board to a DC connector mounted at the board edge. A single horn block with direct machined conical horns (discussed later) will mate to the detector block, aligned with guide pins and secured with fasteners around the outside of the block. For large arrays, individual pixels will be sacrificed in the array to allow for additional fasteners internal to the array footprint to achieve sufficient fastener pressure. Pixel pitch has been chosen at $6 \mathrm{~mm}$. This allows the array size to be kept manageable even at $\sim 1000$ pixels (a $32 \times 32$ array would be $\sim 300 \mathrm{~mm} \times 300 \mathrm{~mm}$ ), while allowing sufficient space to fit all necessary hardware in a single pixel footprint.

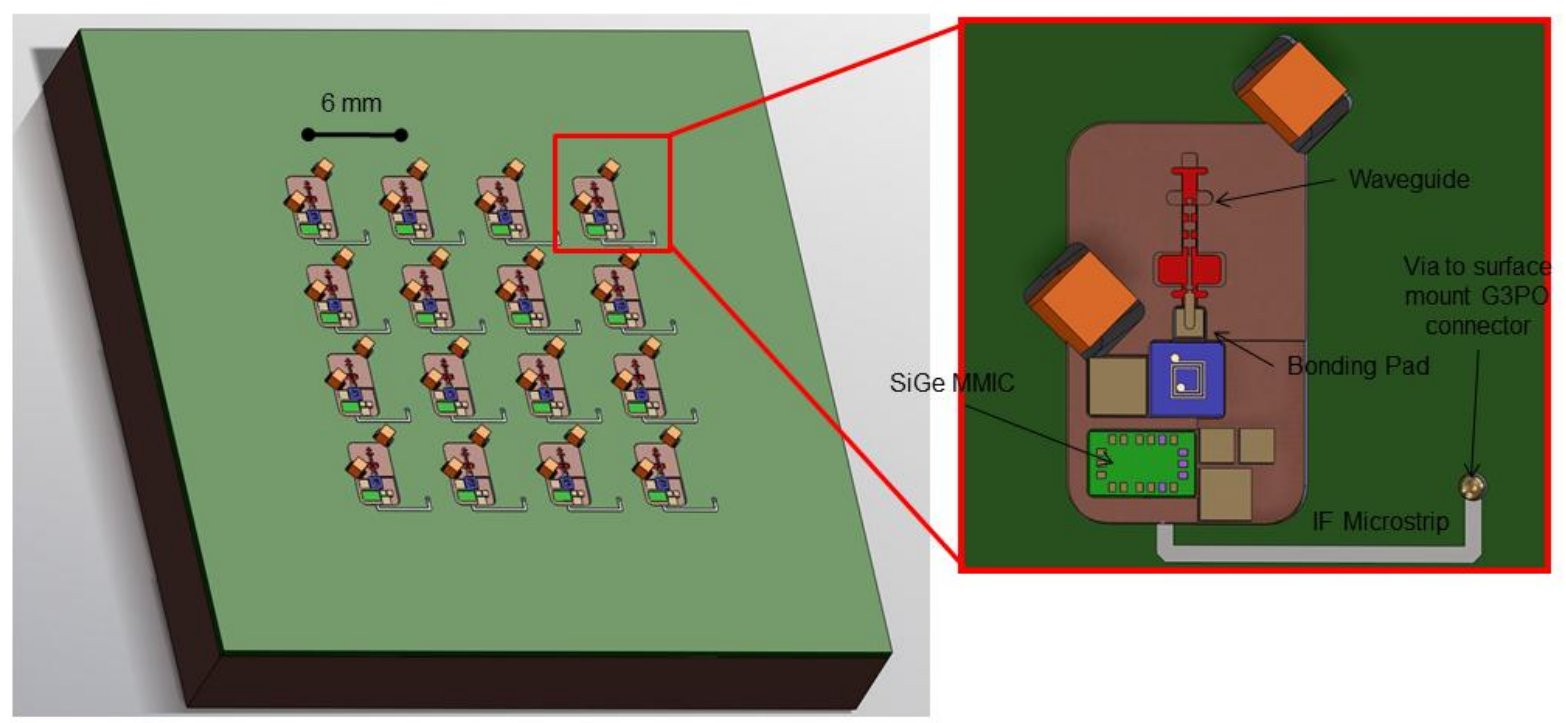

Figure 2: The KAPPa focal plane array architecture. All components of a pixel (waveguide circuit, SIS device, magnet, LNA, IF output and bias components) fit within the $6 \mathrm{~mm} \times 6 \mathrm{~mm}$ pixel pitch. 


\subsection{SIS Devices}

For KAPPa, we have chosen to follow the heritage gained from the SuperCam in designing the discrete SIS device. Our experience from SuperCam has shown that SIS devices fabricated on SOI membrane are robust and easy to mount. We have refined the SuperCam design for this work to improve the ease of mounting and handling while still maintaining optimal performance. We have chosen to baseline a relatively simple single junction design using a traditional $\mathrm{AlO}_{\mathrm{x}}$ barrier. This will maximize chip yield with the relatively high $\mathrm{R}_{\mathrm{n}}$ required to match to the integrated low noise amplifier discussed below. An AlN barrier would provide wider RF bandwidth, but requires very small junction areas for an $\mathrm{R}_{\mathrm{n}} \sim 30 \mathrm{Ohms}$ as desired. This could reduce chip yield to unacceptable levels.

The chip will be fabricated on 5 micron thick SOI membrane. EM simulations have shown that this is the thickest SOI permissible without degradation of performance. The SOI membrane will be patterned to provide mechanical alignment tabs for chip mounting (see figure 3). In the SuperCam device, we used beam leads for this purpose, but we believe using the silicon itself will result in more robust devices. Beam lead tabs with rounded corners will be used for ground and IF contacts. As with SuperCam, the device will use a radial stub waveguide probe with a multi-section RF choke. Unlike SuperCam, the IF and ground are on the same side of the probe, eliminating the need for a meandering high impedance line to serve as a ground return path. This meandering line causes resonances that limit the RF bandwidth. These are tolerable for the lower frequency SuperCam devices, but not for this work. The IF will be brought out on thin film microstrip fabricated on top of the RF choke structure that serves as the ground plane. This potentially reduces chip yield, but has the advantage that it does not require any additional layers in the fabrication.

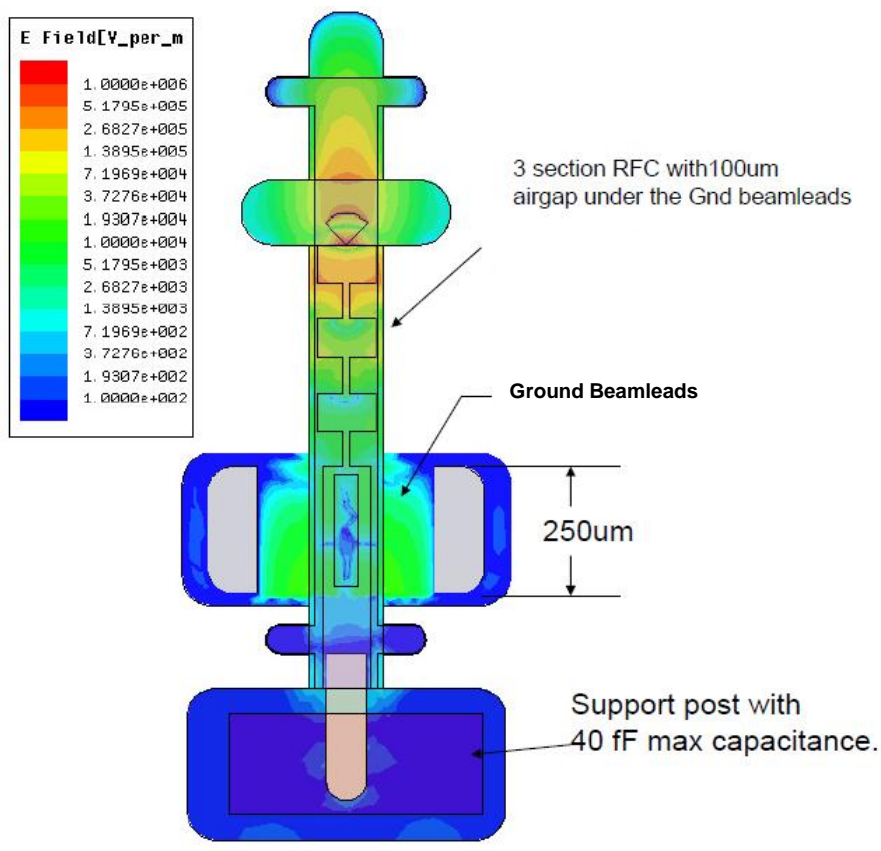

Figure 3: A HFSS simulation of the KAPPa SIS device RF probe and IF output. The device is fabricated on 5 micron thick SOI membrane with beam lead ground and IF connections.

Design of the KAPPa SIS chips were completed in 2011. A batch of test chips (complete chips without the SIS device and RF/IF circuity) were fabricated to allow the development of a handling and assembly procedure that is compatible with large arrays. We found that the KAPPa chips were far easier to handle than the higher aspect ratio SuperCam chips. A procedure was developed to mount the KAPPa device in under 10 minutes. With this procedure, an entire 1024 pixel array could be assembled by a single technician in approximately one month. A picture of a test device mounted in a test fixture using this procedure is shown in figure 4. A IF matching circuit for matching the SIS chip to the SiGe LNA discussed below was carefully designed and refined. It consists only of a single shunt capacitor (used as a bonding stud for the IF beamlead), and a chip inductor. This results in a flat IF response from $0.5 \mathrm{GHz}$ to over $4.5 \mathrm{GHz}$, limited primarily by the IF bandwidth of the SiGe MMIC (section 2.4). 


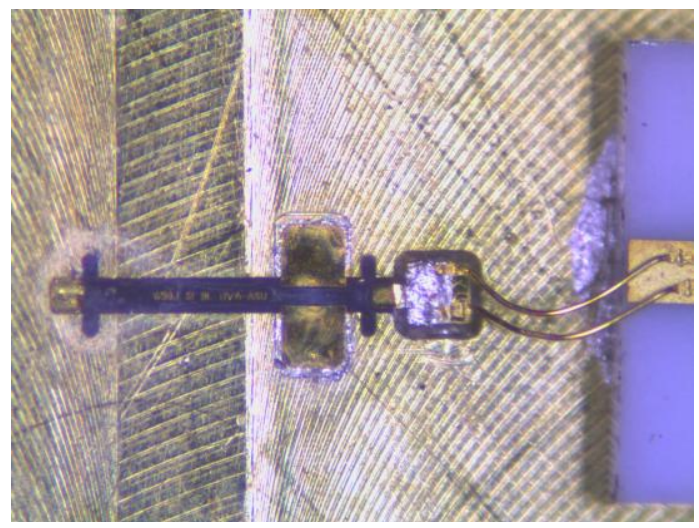

Figure 4: A mechanical model of the KAPPa SIS device mounted in a test fixture. The procedure developed mounts a chip in under 10 minutes.

\subsection{Low Noise Amplifiers}

KAPPa uses a custom designed MMIC amplifier chip integrated directly into each pixel cell of the array. The amplifier is based on Silicon Germanium ( $\mathrm{SiGe}$ ) technology, and has been designed for ultra-low DC power dissipation. SiGe amplifiers have several advantages for integration into large focal plane arrays, including small chip size and good input match (eliminating the need for an isolator). Two designs have been fabricated: One design with an integrated bias tee and another design without the bias tee. This amplifier provides $16 \mathrm{~dB}$ of gain and $<=7 \mathrm{~K}$ noise temperature from 0.5 $4.5 \mathrm{GHz}$ while consuming only $\sim 2 \mathrm{~mW}$ of DC power. Figure 5 shows the chip layout of the device with integrated bias tee, and measured cryogenic performance of the first batch of chips. A slight improvement of noise and bandwidth is possible if $5 \mathrm{~mW}$ of DC power is tolerated.
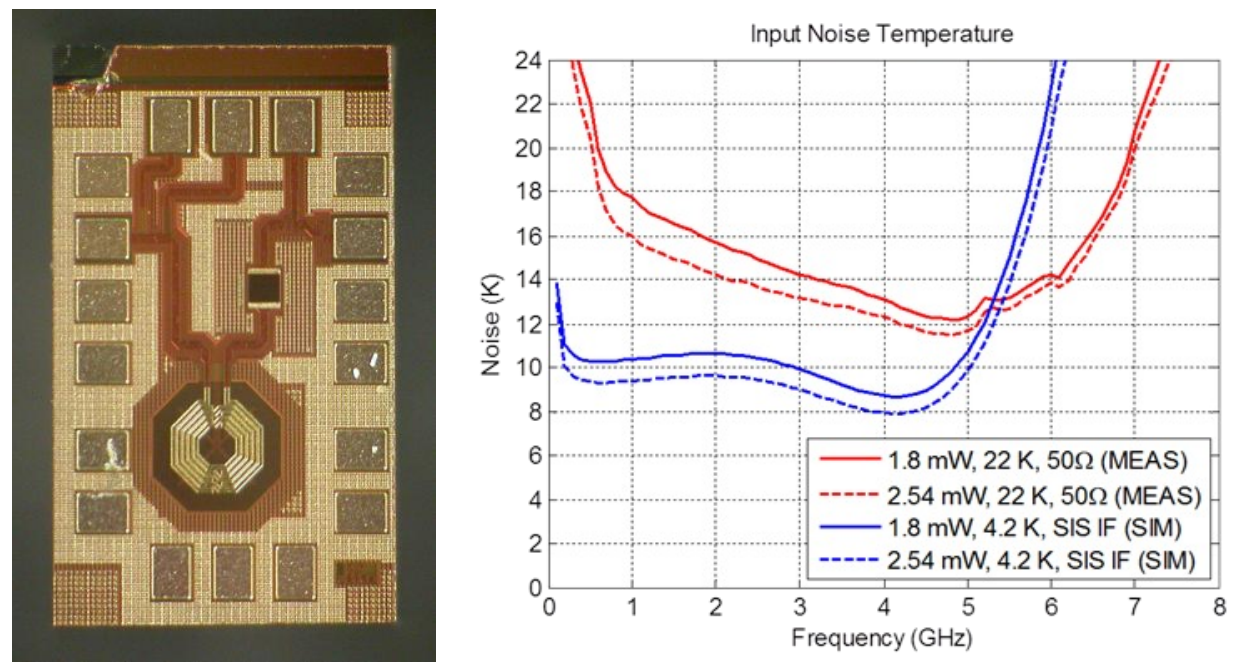

Figure 5: The KAPPa SiGe MMIC chip, $0.5 \mathrm{~mm}$ x $0.8 \mathrm{~mm}$ (left), and measured performance at 1.8 and $2.5 \mathrm{~mW}$ power dissipation into $50 \mathrm{ohms}$, and transformed to the expected SIS IF output impedance.

We have analyzed the integration of this LNA chip into the mixer array. We have chosen to add a simple two component matching network consisting of a surface mount chip inductor in series and a shunt capacitor. This route allows the maximum flexibility to use the chip in the largest number of applications. This results in performance that is not sensitive to SIS device normal resistance from 100-200 ohms. Future generations of this chip could be fabricated to include the matching network (i.e. if a dedicated wafer run is required to populate a large focal plane array). 

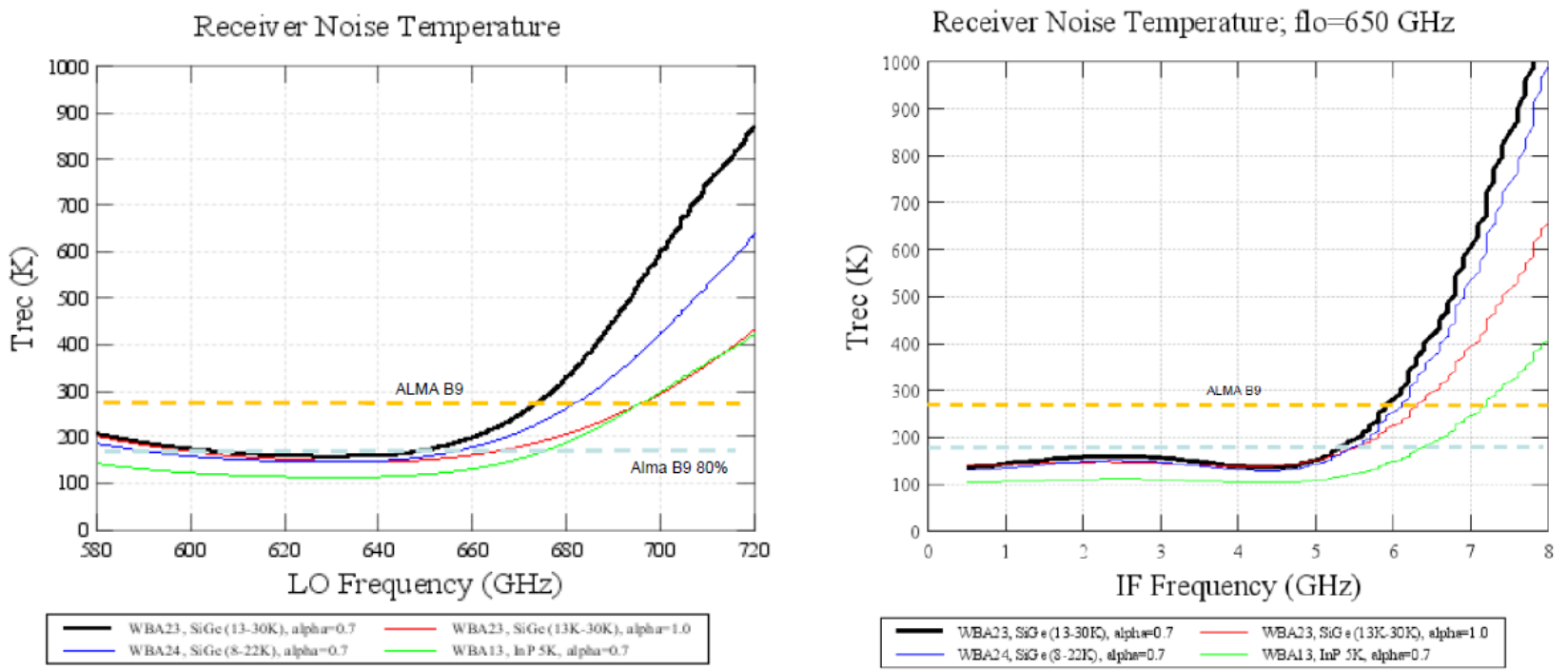

Figure 6: Predicted KAPPa receiver noise temperature as a function of RF frequency (left) and of IF frequency at $660 \mathrm{GHz}$ RF (right). The model includes the measured noise of the WBA23 LNA, and assumes 5\% optical losses in front of the mixer.

Testing of Low Noise Amplifiers for KAPPa have been completed. The amplifier works as expected, with the exception of slightly higher noise than designed $(9 \mathrm{~K}$ vs. $7 \mathrm{~K}$, see figure 5$)$. This increased noise has a very small effect on the instrument's final system noise temperature. The performance of the system (including the SIS device, LNA and expected losses) has been carefully modeled and is summarized in figure 6. We find that KAPPa should meet the ALMA band 9 specifications for receiver noise temperature for the majority of the band, only failing at the highest frequencies (above $700 \mathrm{GHz}$ ) due to our use of $\mathrm{Nb}$ superconductor, resulting in increased conversion loss at the upper end of the band. This could be mitigated through the use of NbTiN/Al films, but we decided against this complication at this stage in favor of the highest possible chip yield. Additional work is proceeding on the LNA MMICs, attempting to reduce the noise in future runs to (or below) the design value.

\subsection{Permanent magnets}

A permanent magnet has been designed using iron concentrators and a very small commercially available rare earth magnet. This magnet produces enough field to reach past the third minimum in Josephson current for our junction size. Having a large field makes reaching precisely the right field level less critical because the magnitude of the Josephson current peaks decrease with increasing magnetic field. This small magnet easily fits in the $6 \mathrm{~mm}$ pixel pitch. A CAD model of this magnet and the predicted field between the concentrators is shown in figure 7 .
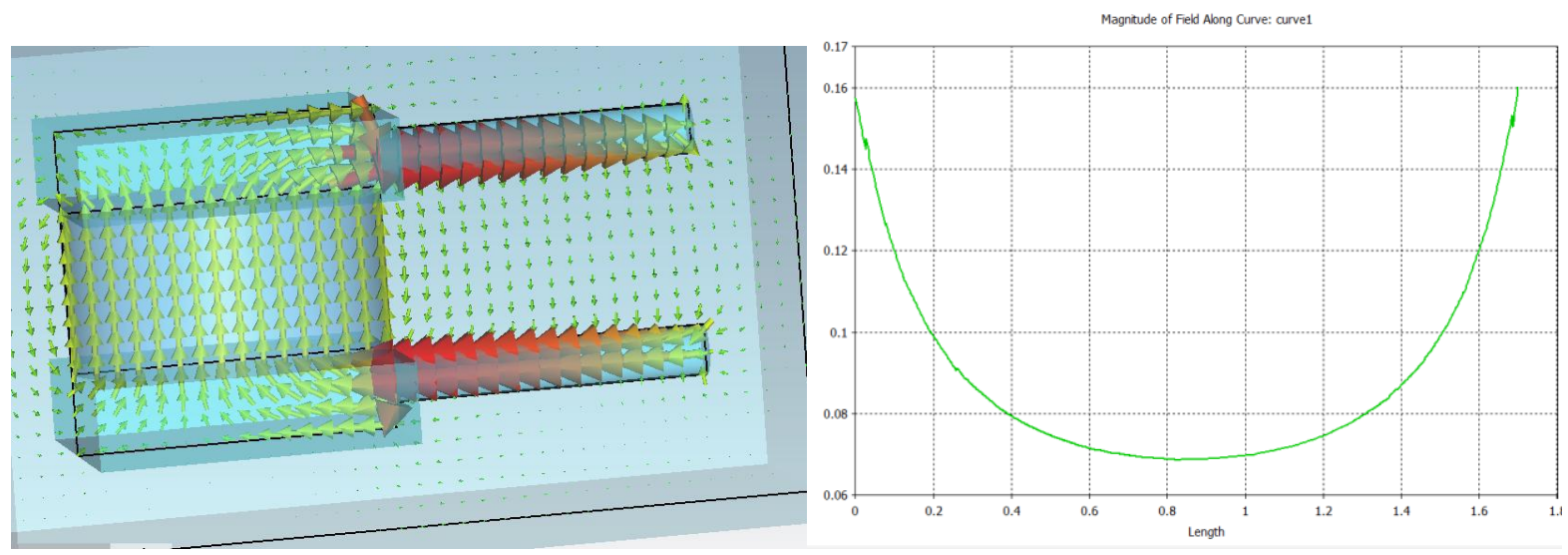

Figure 7: The KAPPa permanent magnet (left) and magnetic field (in Tesla) as predicted by the CST EM Studio magnetostatic modeling package between the concentrators at the junction plane (right). 


\subsection{Local Oscillator and Optics}

A local oscillator has been purchased from Virginia Diodes Inc. covering the $650-700 \mathrm{GHz}$ band with $1-2 \mathrm{~mW}$ of available power. This is certainly adequate for pumping 16 pixels. A beam forming phase grating and a Mylar beamsplitter will be used to spatially multiplex and then inject the LO with high efficiency into the detector array. The LO level will then be measured to determine the necessary drive power for $\sim 1000$ pixels. The phase grating LO multiplexing scheme was chosen as the most amicable to scaling to 1000 pixels. Waveguide 2D multiplexer schemes will become unwieldy at this pixel count. Integrating couplers or the multipliers themselves in the mixer array will dramatically increase the complexity of the focal plane. One option is a 2D array of individual multipliers, but this alone is a significant development effort.

The array will incorporate a 3 segment profiled horn designed by collaborators at Oxford University [9]. This horn can be drilled with a relatively simple single flute milling cutter. It offers beam quality, cross-polarization and bandwidth comparable to a corrugated horn while being simple to fabricate. The horn has been designed with an aperture of $6 \mathrm{~mm}$, producing a beam of the desired $\mathrm{f}$ number ( $\mathrm{f} / 6.5$, identical to SuperCam) with no additional cold optics required. This dimension ultimately drives the pixel pitch for the KAPPa array. Finer pixel pitch could be achieved with faster feed optics. The use of a directly machined profiled horn dramatically simplifies the design of a large array in several ways. The large f-number horns produce a beam that can directly couple to warm optics (in this case the SuperCam warm optical system,) eliminating the need for individual cold mixer lenses or mirrors. The profiled horn is also straightforward to fabricate, so the production of a 1000 element horn array by direct machining becomes tractable. Custom drilling tools that will be used to produce this horn and a conical taper rectangular-to-circular transformer are in production now. The horn profile and predicted beam pattern are shown in figure 8 . Transition to oval waveguide is provided by a 13 degree opening angle conical taper. This allows the transformer to be fabricated at the same time as the horn with a conical drilling tool. The oval waveguides are machined in the backside of the horn block using an ultra-high precision milling machine with a high accuracy touch probe system to ensure alignment [10].
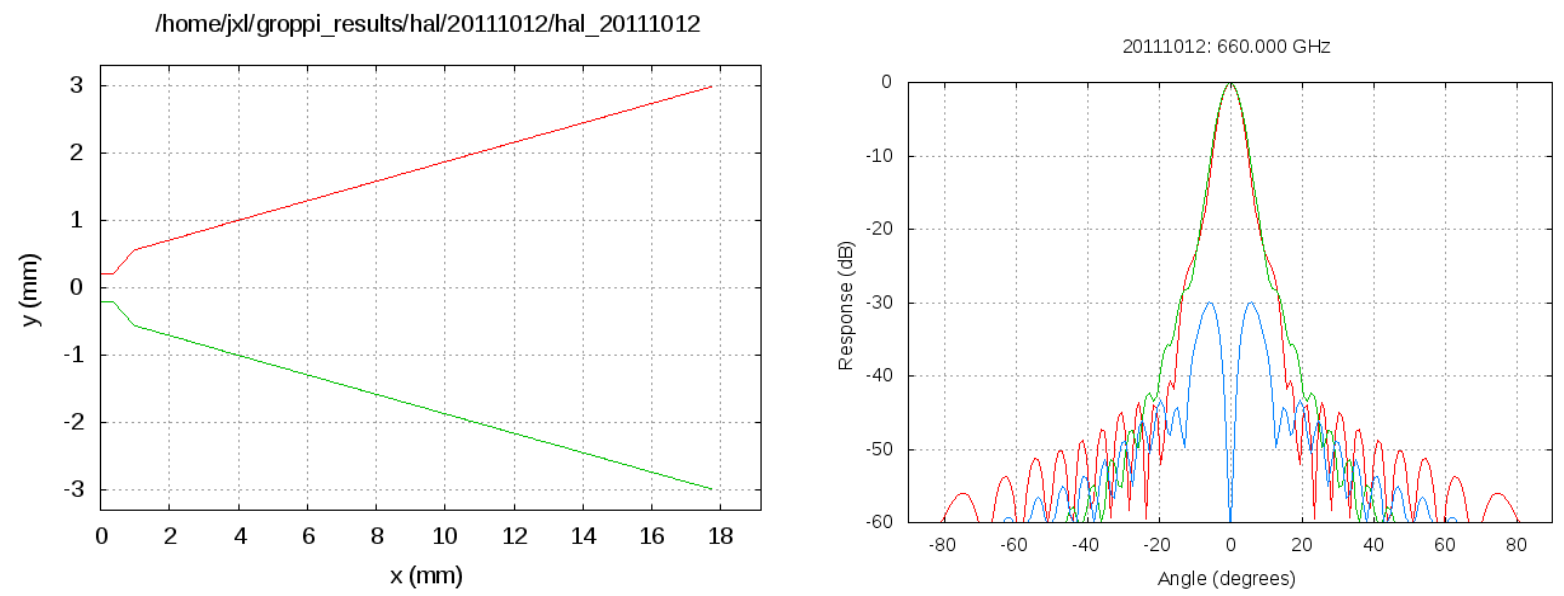

Figure 8: Horn profile of the KAPPa conical horn (left), and predicted beam pattern at $660 \mathrm{GHz}$ (right). The E and $\mathrm{H}$ plane copular patterns are shown in green and red. The crosspolar pattern cut at 45 degrees is shown in blue.

\subsection{IF Flex Circuits}

Arrays with $\sim 1000$ pixels will require a new method of IF signal transmission. All current arrays, including SuperCam, use individual semi-rigid stainless steel coaxial cables for each IF signal. Larger format arrays will require some sort of multi-conductor IF interconnect to simplify the electrical and cryogenic design of the system. We have produced a multichannel flex circuit design based on stripline with transitions to microstrip at each end that meet the needs of KAPPa. We have chosen stripline to minimize RFI, allow routing and heatsinking inside the cryostat without interfering with fields on the transmission line, and to allow potting of the flex circuit in a vacuum flange without the need for connectors. The transition to microstrip at the ends allows for straightforward connection to other circuits. The design uses Rogers Ultralam 3000 flexible RF circuit material with thin copper cladding (see figure 9). We have determined that the heat load presented by the metallization is suitable for cryogenic applications. This circuit has a loss of $4.5 \mathrm{~dB}$ per meter, and a heat load of $5 \mathrm{~mW}$ when heat sunk at $15 \mathrm{~K}$ (150 mm from the $4 \mathrm{~K}$ connection). The circuit is $10 \mathrm{~mm}$ 
wide in the stripline center section, widening to $18 \mathrm{~mm}$ at the stripline to microstrip transition. We have been working with a circuit fabrication company specializing in Ultralam 3000 circuits to ensure the design is compatible with the fabrication process. Connection to the focal plane and warm IF system will be completed using surface mount Corning Gilbert G3PO press-on connectors mounted on the flex circuit or by direct wire bonding.

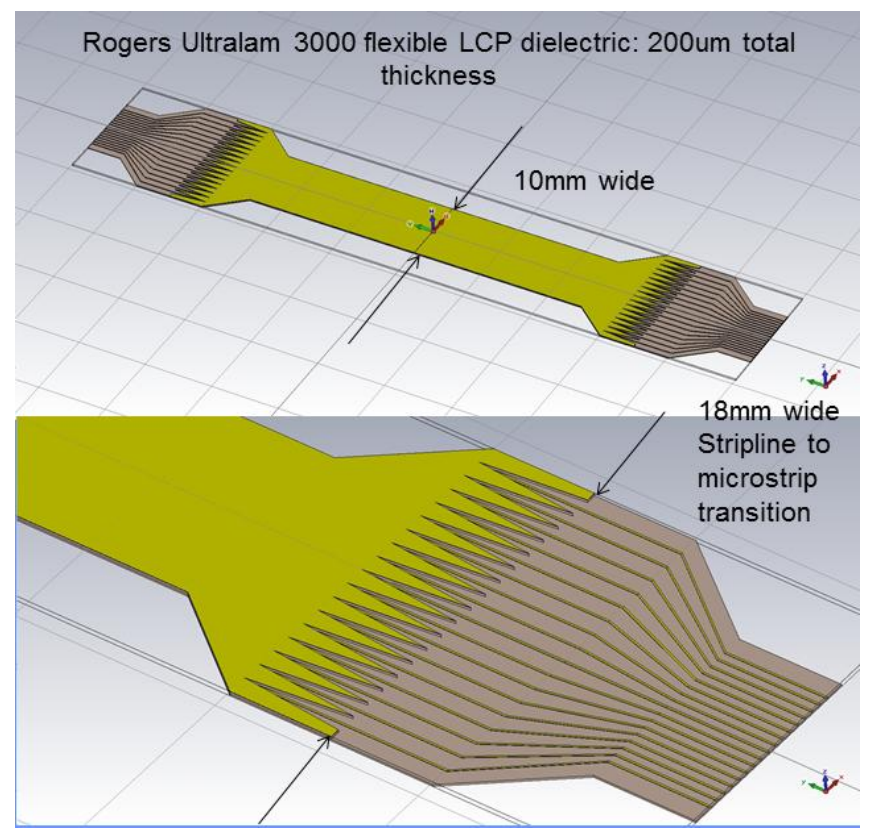

Figure 9: A CST Microwave Studio model of the 16 channel IF flex circuit designed for KAPPa.

\section{SINGLE PIXEL TEST MIXER}

A single pixel test mixer has been designed (See figure 10) to examine the performance of all the components of the KAPPa pixel cell in detail before moving on to the construction of the 16 pixel $2 \mathrm{D}$ array. This single pixel mixer contains a single pixel cell identical to the design envisioned for the array, in a block that can accommodate either a traditional electromagnet or our newly designed permanent magnet (see below). The test mixer uses a traditional SMA connector rather than a Corning Gilbert G3PO connector for simplicity. The Corning Gilbert connector has already been proven to work at cryogenic temperatures with SuperCam. The magnet assemblies can be exchanged without opening the block, allowing comparison of just the effect of the magnet using the identical SIS device and waveguide circuit.

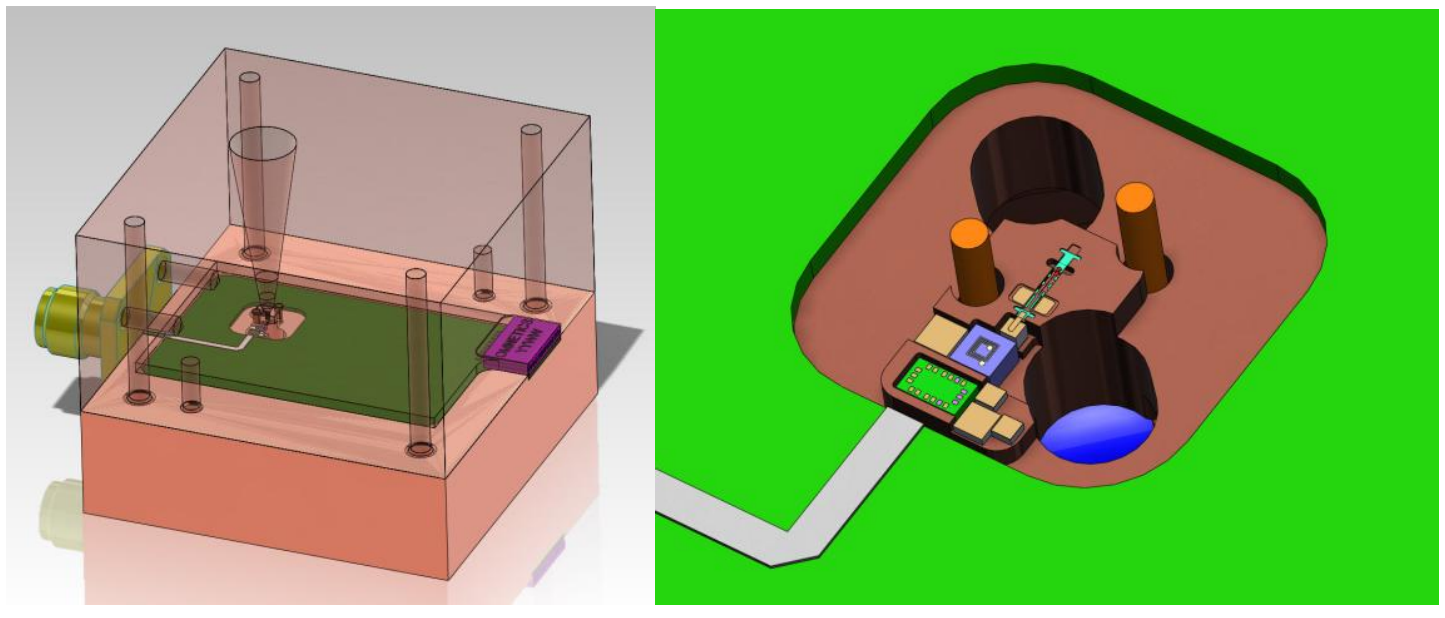

Figure 10: A CAD model of the KAPPa single pixel test mixer (left) and a closeup of the pixel cell containing all mixer components in a $6 \mathrm{~mm} \times 6 \mathrm{~mm}$ pixel footprint. 


\section{CONCLUSION}

The KAPPa project is designing a 16 pixel pathfinder focal plane array for the $660 \mathrm{GHz}$ atmospheric window to develop and test the technologies necessary to construct coherent arrays with $\sim 1000$ pixels. The project has identified several issues with scaling current array technologies to this pixel count. KAPPa will surmount these challenges using 2D integration of SIS mixers, SiGe MMIC low noise amplifiers and permanent magnets, keeping the design of each component as simple as possible to maximize pixel count and yield. All critical KAPPa components have been designed, and are now in fabrication or have completed testing. We are building a single pixel test mixer to validate the design of the KAPPa mixer cell. Once this is complete, we will make any necessary design refinements and produce the 16 pixel focal plane unit.

\section{REFERENCES}

[1] Poglitsch, A. et al. "Herschel Photodetector Array Camera and Spectrometer," Proc. SPIE, 7010, 4, (2008).

[2] Rieke, G., et al., "The Multiband Imaging Photometer for Spitzer", ApJSS, 154, 25, (2004).

[3] Audley, A., et al. "An update on the SCUBA-2 project", Proc. SPIE, 5498, 63, (2004).

[4] Groppi, C., et al., "Testing and Integration of Supercam, a 64-Pixel Array Receive for the $350 \mathrm{GHz}$ Atmospheric Window," Proc. SPIE 7741, 774110X, (2010).

[5] Goldsmith, P., et al., "Coherent Detector Arrays for Millimeter and Submillimeter Astronomy," Astro2010: The Astronomy and Astrophysics Decadal Survey, Technology Development Papers, no. 11, (2009).

[6] Kerr, A., et al., "In Support of Instrument Technology Development for THz Astronomy," Astro2010: The Astronomy and Astrophysics Decadal Survey, Technology Development Papers, no. 29, (2009).

[7] Goldsmith, P., et al., "Large-Scale Structure of the Molecular Gas in Taurus Revealed by High Linear Dynamic Range Spectral Line Mapping”, Astrophysical Journal, vol. 680, pp. 428-445, (2008).

[8] Narayanan, G., et al., "The Five College Radio Astronomy Observatory CO Mapping Survey of the Taurus Molecular Cloud", Astrophysical Journal Supplement Series, vol. 177, pp. 341-361, (2008).

[9] Leech, J., et al., "Experimental Investigation of a Low-Cost, High Performance Focal-Plane Horn Array," IEEE Trans. THz Science \& Technology, v. 2, no. 1, (2012).

[10] Groppi, C., et al., "Automated CNC Micromachining for Integrated THz Waveguide Circuits," $21^{\text {st }}$ International Symposium on Space Terahertz Technology, http://www.nrao.edu/meetings/isstt/2010.shtml, (2010). 